\title{
PLEURAL TUBERCULOSIS IN A PATIENT AFTER COVID-19 PNEUMONIA
}

\author{
Sevil ALKAN ${ }^{1}$, Emine Kübra DINDAR DEMIRAY²*, Mehmet DURĞUN ${ }^{3}$, Özgür PAŞA ${ }^{4}$, Sinem SEFER ${ }^{5}$
}

\author{
${ }^{1}$ Çanakkale Onsekiz Mart University, Faculty of Medicine, Department of Infectious Diseases and Clinical Microbiology, 17020, \\ Çanakkale, Turkey \\ ${ }^{2}$ Bitlis Tatvan State Hospital, Department of Infectious Diseases and Clinical Microbiology, 13200, Tatvan, Bitlis, Turkey \\ ${ }^{3}$ Bitlis State Hospital, Department of Chest Diseases and Tuberculosis, 13000, Bitlis, Turkey \\ ${ }^{4}$ Bitlis Tatvan State Hospital, Department of Microbiology, 13200, Tatvan, Bitlis, Turkey \\ ${ }^{5}$ Çanakkale Onsekiz Mart University, Faculty of Medicine, 17020, Çanakkale, Turkey
}

\begin{abstract}
During the coronavirus disease 2019 (COVID-19) pandemic, it was observed that the clinics of existing and undiagnosed tuberculosis (TB) patients were more severe, especially in low and middle-income countries where TB is endemic and there is a lack of healthcare. There is limited literature on whether the coexistence of TB and COVID 19 or the lung damage/immune state caused by COVID-19, or whether immunosuppressive therapies used during the treatment of COVID-19 whether it creates a predisposition to TB. In this case report, we aimed to present a 66-year-old female patient diagnosed as pleural tuberculosis after COVID-19 pneumonia and to examine the possible relationship between COVID-19 and TB.
\end{abstract}

Keywords: COVID-19, Pleural tuberculosis, Tuberculosis, Empyema

\begin{tabular}{|c|c|c|}
\hline \multicolumn{3}{|c|}{$\begin{array}{l}\text { *Corresponding author: Bitlis Tatvan State Hospital, Departm } \\
\text { E mail: e.kubradindar@hotmail.com (EK DINDAR DEMIRAY) }\end{array}$} \\
\hline Sevil ALKAN & (iD) https://orcid.org/0000-0003-1944-2477 & Received: May 29, 2021 \\
\hline Emine Kübra DINDAR DEMIRAY & https://orcid.org/0000-0001-6459-7182 & Accepted: October 07, 2021 \\
\hline Mehmet DURĞUN & https://orcid.org/0000-0002-1289-7500 & Published: January 01, 2022 \\
\hline Özgür PAŞA & https://orcid.org/0000-0002-3137-545X & \\
\hline Sinem SEFER & (iD) https://orcid.org/0000-0001-8209-1978 & \\
\hline
\end{tabular}

\section{Introduction}

After the first case was detected in December 2019, Corona virus 2019 (COVID-19) spread all over the world and the first case in our country was announced on 11 March 2020. In 1, April 2020 that the case across the country the Republic of Turkey (TC) is defined by the Ministry of Health. As of March 4, 2021, 2.734 .836 infected with COVID-19 and 28.771 deaths have been reported in our country. Our country is the 7th country with the most COVID-19 cases in the world according to the data of March 4, 2021. (URL 1; URL 2).

According to our Ministry of Health's statistics, the incidence of tuberculosis (TB) cases has decreased from 29.4 percent in 2005 to 14.1 percent in 2018 , but TB cases continue to be seen throughout the country (URL 3).

In this case report; we aimed to examine the possible relationship between COVID-19 and TB by presenting a 66-year-old female case diagnosed with pleural TB, which could possibly be overlooked due to the intensity during the pandemic. In the literature available, it was found that there are quite a limited number of research articles and case presentations on this subject. Therefore, we think that the presented case will contribute to the literature.

\section{Case Report}

A 66-old female patient with complaints of dispne, fever and cough was referred to our hospital from an external center with a pre-diagnosis of COVID-19 pneumonia. Her general condition was moderate, conscious, cooperative and oriented. Fever $38{ }^{\circ} \mathrm{C}$, heart rate $81 / \mathrm{min}$, blood pressure $120 / 80 \mathrm{mmHg}$. There was no obvious pathology in respiratory sounds. Other system examinations were normal. In our center, the patient, whose swab samples were taken from the oropharynx and nasopharynx, was hospitalized upon the detection of Thorax Computed Tomography (CT) compatible with viral pneumonia (ground glass opacity in one area). The patient was given favipravir $200 \mathrm{mg}$ tablets $(2 \times 1600 \mathrm{mg}$ loading dose, $2 \times 600 \mathrm{mg}$ maintenance dose), low molecular weight heparin, and dexamethasone $6 \mathrm{mg} /$ day treatments by obeying the proposal of the Turkish Ministry of Health's COVID-19 Diagnosis and Treatment Guide. The patient received a total of dexamethasone $6 \mathrm{mg} /$ day treatment for a total of 5 days. The patient's COVID-19 RT-PCR test was positive. The patient whose general condition improved after 7 days of hospitalization she was discharged.

The patient was admitted again 15 days after discharge with severe shortness of breath and inability to lie flat. 
The patient was reintroduced. In the thoracic CT of the patient, right pleural effusion (empyema?) and fluid collection in the major fissure and cardiothoracic ratio was greater than $50 \%$. Parenchymal infiltration was not detected (Figure 1). In laboratory examinations; White blood cell count was $4700 / \mathrm{mm}^{3}$, hemoglobin level was $11 \mathrm{~g} / \mathrm{dL}$, and thrombocyte count was $187000 / \mathrm{mm}^{3}$. In biochemical examination; albumin level was $3.3 \mathrm{~g} / \mathrm{dL}$, aspartate aminotransferase (AST) level was $45 \mathrm{U} / \mathrm{L}$, alanine aminotransferase (ALT) level was $41 \mathrm{U} / \mathrm{L}$. Among the acute phase reactants, the erythrocyte sedimentation rate (ESR) was $82 \mathrm{~mm} /$ hour and the C reactive protein (CRP) level was $13.6 \mathrm{mg} / \mathrm{dL}$. At the second day of the patient's hospitalization, she had a fever, reaching $38.2{ }^{\circ} \mathrm{C}$ especially at night. Cough and sputum symptoms are also added to his symptoms. COVID-19 PCR test was taken again. It was negative. There was no growth in sputum, blood and urine cultures. Ehrlich-Ziehl-Neelsen (EZN) staining sent from sputum three times resulted in negative. Brucella (Rose Bengal), anti HIV, anti HCV, VDRL, HbsAg test results were negative for differential diagnosis. Abdominal ultrasonography (USG) was requested, no abnormality was dedected.

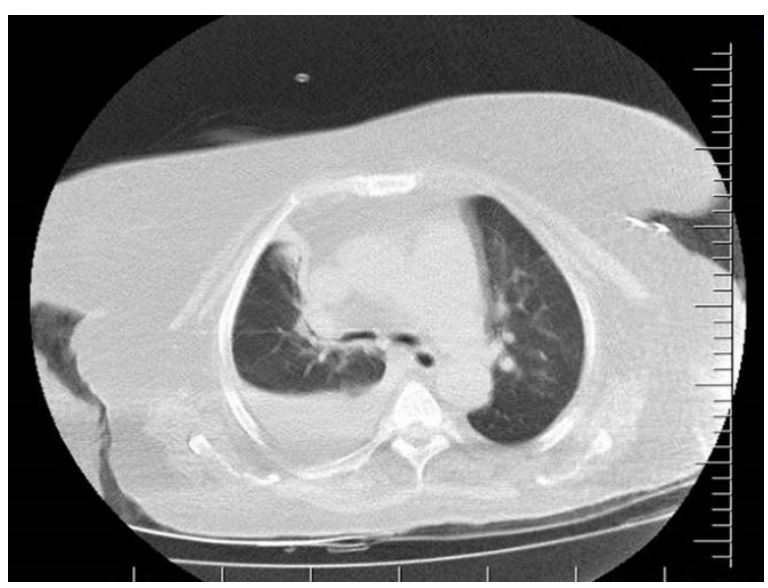

Figure 1. Thoracic CT of the patient shows right pleural effusion (empyema?).

Chest diseases consultation was requested from the patient and diagnostic thoracentesis was performed. Aerobe + anerobic cultures from the pleural fluid, EZN staining, PCR (Polymerase Chain Reaction), sample for biochemical examinations and cytological examination were sent from the patient. Albumin level was $2.88 \mathrm{~g} /$ $\mathrm{dL}$, glucose level was $64 \mathrm{mg} / \mathrm{dL}$, white blood cell count was $2250 / \mathrm{mm}^{3}$ in the pleural fluid taken, but bacteria were not seen in Gram staining. There was lymphocyte dominance in Giemsa staining, and in EZN staining, administration was detected as +1 acid-resistant basil (ARB) positivity in pleural fluid (Figure 2). Simultaneous serum albumin level was determined as $3.3 \mathrm{~g} / \mathrm{dL}$. Serum pleural fluid albumin gradient was determined as 0.42 ( $<1.2$ exudate) and was interpreted as exudate according to Light's criteria. Lymphocyte predominance was found in pleural fluid cytological examination. ADA level and Quantiferon Gold test could not be sent. The pleural fluid TB PCR result sent and found as a positive. Since the patient's clinic was compatible with $\mathrm{TB}$, antituberculosis treatment with +4 (isoniazid $5 \mathrm{mg} / \mathrm{kg} /$ day, rifampicin $10 \mathrm{mg} / \mathrm{kg} /$ day, pyrazinamide $25 \mathrm{mg} / \mathrm{kg} /$ day, ethambutol $25 \mathrm{mg} / \mathrm{kg} /$ day) was started. Her fever started to regress 3 days after the start of treatment. The patient recovered from clinical and laboratory findings after five days of fever-free follow-up. It was found that the patient was completely regressed under the control of the pleura at the 2 nd month control outpatient clinic.

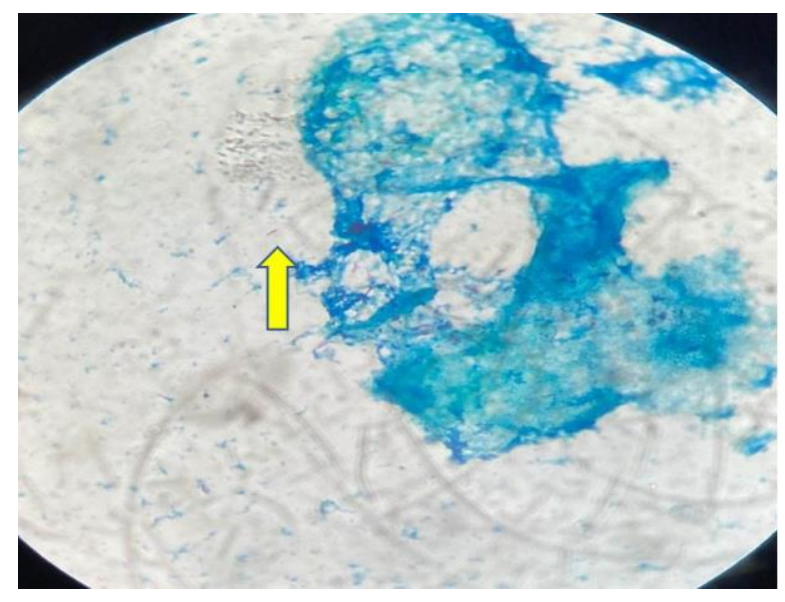

Figure 2. +1 acid-resistant basil (ARB) positivity in pleural fluid.

\section{Results and Discussion}

There is limited literature information on the relationship between COVID-19 and tuberculosis (TB). Recent studies have shown that individuals with latent or active ТВ may be more susceptible to COVID-19, and disease progression caused by this virus may be faster and more severe (URL 4). Tadolini et al. (2020) coexistence of COVID-19 and TB was examined in a cohort study consisting of 49 patients. In this study; it has been reported that $53 \%$ of the patients had a history of TB in the past, $18.3 \%$ of them were simultaneously diagnosed with TB and COVID-19, and $38.8 \%$ of TB patients had COVID-19 during TB treatment.

During the pandemic, there can be severe consequences for existing and undiagnosed TB patients, especially in low- and middle-income countries where TB is endemic and healthcare is inadequate. In addition, the fact that healthcare workers, who were previously involved in the fight against TB, had to focus on patients diagnosed with COVID 19 globally may also contribute to the increase in the number of overlooked TB cases or treatment failure (URL 4; Tadolini et al., 2020; Togun et al., 2020).

COVID-19 often causes severe pneumonia and possibly long-term lung damage in the elderly and / or those with underlying comorbidities. There is not enough consensuses about the long-term effect of this virus on lung function, which is only a year old. Potential interactions between TB and COVID 19 pneumonia seem 
likely. Being diagnosed with COVID-19 cannot rule out the underlying TB. Therefore, careful examination is required (Tadolini et al., 2020; Togun et al., 2020; Wingfield et al., 2020).

Mousquer et al. (2020) reported that in a review study; from an immunological standpoint, in TB / COVID-19 coinfection, disorders caused by immunomodulation by each pathogen tend to cause an unstable inflammatory response that can promote the progression and worsening of both diseases. In this study, PUBMED database has been examined and as a summary; it has been argued that both active TB and a previous TB history are an increased risk for the development of COVID-19 and may lead to a worsening of the COVID 19 prognosis (Mousquer et al., 2020). Pleural tuberculosis usually occurs when a subpleurally located caseous focus in the lung opens into the pleural space (Tadolini et al., 2020; Togun et al., 2020; Wingfield et al., 2020). The presented case did not have any finding suggestive of TB at the first admission. However, the patient who presented with pleural effusion after discharge was diagnosed with pleural TB. However, it could not be determined whether this disease occurred due to possible steroid use or immunosuppression caused by COVID-19. In addition, there was no known history of TB in presented case, in addition in her family or close contacts.

There are limited number of TB COVID-19 co-infection cases in the literature (Tadolini et al., 2020; Khurana et al., 2020; AlKhateeb et al., 2020; Motta et al., 2020). However, TB empyema and COVID-19 infection reports similar to the presented patient have not been found in the literature.

In a cohort review of 69 cases investigating COVID-19 and TB co-infections, Motta et al. (Motta et al., 2020) reported that mortality is more likely in elderly patients with comorbidities, and that mortality rates are higher in young people in infections caused by drug-resistant Myobacterium tuberculosis strains. Although the present case was 66 years old, no mortality developed and no growth occurred in the patient's TB culture.

\section{Conclusion}

In summary, despite the fact that our increased workload during the pandemic, it has become important to be more extremely vigilant about TB in our country, which is endemic for the disease, particularly in cases of pleural effusion or clinical suspicion, and to include TB in the differential diagnosis. Since there is frosted glass in one area, it was premature for me to administer steroids. TB may be activated due to immunosuppression.

\section{Author Contributions}

Authors have obtained the necessary data by evaluating our case in detail Conflict of Interest: The authors declare that there is no conflict of interest.

\section{Conflict of Interest}

The authors declare that there is no conflict of interest.

\section{Ethical Approval/Informed Consent}

Written an informed consent form was obtained from the patients for the case presentation and necessary information was given to the family. After fully explaining the purpose of the study to the patient, consent was obtained for the study and case presentation.

\section{References}

AlKhateeb MH, Aziz A, Eltahir M, Elzouki A. 2020. Bilateral footdrop secondary to axonal neuropathy in a tuberculosis patient with co-infection of COVID-19: A Case Report. Cureus, 12(11), e11734. https://doi.org/10.7759/cureus.11734.

Khurana AK, Aggarwal D. 2020. The (in) significance of TB and COVID-19 co-infection. Eur Respir J, 56(2), 2002105. https://doi.org/10.1183/13993003.02105-2020.

Motta I, Centis R, D'Ambrosio L, García-García JM, Goletti D, Gualano G, Lipani F, Palmieri F, Sánchez-Montalvá A, Pontali E, Sotgiu G, Spanevello A, Stochino C, Tabernero E, Tadolini M, van den Boom M, Villa S, Visca D, Migliori G.B. 2020. Tuberculosis, COVID-19 and migrants: Preliminary analysis of deaths occurring in 69 patients from two cohorts. Pulmonol, 26(4): 233-240.

Mousquer GT, Peres A, Fiegenbaum M. 2021. Pathology of TB/COVID-19 Co-Infection: The phantom menace. Tuberculosis, 126: 102020.

Tadolini M, Codecasa LR, García-García JM, Blanc FX, Borisov S, Alffenaar JW, Andréjak C, Bachez P, Bart PA, Belilovski E Cardoso-Landivar J, Centis R, D'Ambrosio L, Luiza De SouzaGalvão M, Dominguez-Castellano A, Dourmane S, Jachym MF, Froissart A, Giacomet V, Goletti D, Grard S, Gualano G, Izadifar A, Le Du D, Royo MM, Mazza-Stalder J, Motta I, Ong CWM, Palmieri F, Rivière F, Rodrigo T, Silva DR, Sánchez-Montalvá A, Saporiti M, Scarpellini P, Schlemmer F, Spanevello A, Sumarokova E, Tabernero E, Tambyah PA, Tiberi S, Torre A, Visca D, Murguiondo MZ, Sotgiu G, Migliori GB. 2020. Active tuberculosis, sequelae and COVID-19 co-infection: first cohort of 49 cases. Eur Respir J, 56(1): 2001398.

Togun T, Kampmann B, Stoker NG, Lipman M. 2020. Anticipating the impact of the COVID-19 pandemic on TB patients and TB control programmes. Ann Clin Microbiol Antimicrob, 19(1): 21.

URL 1. https://covid19.saglik.gov.tr/ (access date: January 31, 2021).

URL 2. https://www.who.int/emergencies/diseases/novelcoronavirus-2019 (access date: March 4, 2021).

URL 3. https://hsgm.saglik.gov.tr/tr/tuberkuloz-istatistikler (access date: March 4, 2021).

URL 4. Liu Y, Bi L, Chen Y, Wang Y, Fleming J, Yu Y, et al. Active or latent tuberculosis increases susceptibility to COVID-19 and disease severity. MedRxiv. [preprint]. https://www.medrxiv.org/content/10.1101/2020.03.10.200 33795v1 (access date: March 4, 2021).

Wingfield T, Cuevas LE, MacPherson P, Millington KA, Squire SB. 2020. Tackling two pandemics: a plea on World Tuberculosis Day. Lancet Respir Med, 8(6): 536-538. 